\title{
Proper orthogonal decomposition investigation in fluid structure interaction
}

\author{
Erwan Liberge $^{*, * *}$ - Mustapha Benaouicha*,** - Aziz Hamdouni* \\ * LEPTAB, Université de la Rochelle, Avenue Michel Crépeau \\ F-17042 La Rochelle cedex 1 \\ ** EIGSI, 26, rue de Vaux de Foletier \\ F-17041 La Rochelle cedex 1 \\ erwan.liberge@univ-lr.fr
}

\begin{abstract}
This paper describes Reduced Order Modeling (ROM) in Fluid Structure Interaction (FSI) and discusses Proper Orthogonal Decomposition (POD) utilization. The ROM method was selected because its performance in fluid mechanics. The principal problems of its application in FSI are due the space character of the modes resulting from the POD whereas domains are mobile. To use POD in moving domain, a charateristic function of fluid is introduced in order to work on a fixed rigid domain, and the global velocity (fluid and structure) is studied. The POD modes efficiency is tested to reconstruct velocity field in one and two-dimensional FSI case. Then reducing dynamic system using POD is introduced in moving boundaries problem. In addition, the one dimensional case of Burgers equation coupled with spring equation is tested.

RÉSUMÉ. Dans cet article nous nous intéressons à la réduction de modèle en interaction fluide structure (IFS) et plus particulièrement à l'application de la méthode de décomposition orthogonale aux valeurs propres (POD). Le choix de cette méthode fait suite aux performances de son application dans le domaine de la mécanique des fluides. La problématique principale de son application en IFS est due au caractère spatial des modes issus de la POD alors que les domaines sont mobiles. Afin d'y rémédier, une fonction caractéristique propre au domaine fluide, permettant ainsi de traiter globalement le problème sur un domaine fixe a été introduite. Les performances de la POD via cette méthode ont été testées sur un cas monodimensionnel et un cas bidimensionnel. La méthode de réduction du système dynamique est ensuite appliquée au cas monodimensionnel d'un fluide dans un piston.
\end{abstract}

KEYWORDS: POD, fluid structure interaction, moving boundaries, reduced model. MOTS-CLÉS : POD, interaction fluide structure, réduction de modèle.

DOI:10.3166/REMN.16.401-418(C) 2007 Lavoisier, Paris. Tous droits réservés

REMN - 16/2007. Fluid structure interaction, pages 401 to 418 


\section{Introduction}

Although numerical tools are more and more performant that makes a good simulations possible for fluid structure interaction, data storage and computational time are still the two major limiting factors.

Constructing a Reduced-Order Model (ROM), in order to reduce the size of the model and the computational cost and also obtaining good simulation, is essential in this domain. Different methodologies had been proposed, the most significant are refered by (Dowell et al., 2001).

There are two possible ways to construct ROM. The most famous one uses the notion of eigenmodes. This approach characterizes a field in terms of a relatively small number of global modes. By modes we mean a distribution of variables that characterizes a gross motion of the physical system studied. There are several techniques to find these modes. One of the technique concentrates on extract the eigenmodes from the model used (Romanowski et al., 1996; Mahajan et al., 1994). However, in case of very high-dimensional system, extracting eigenmodes can be very computationally expensive. Thus, we use another methodology like balanced modes (Baker $e t$ al., 1996; Rule et al., 2000) or Proper Orthogonal Decomposition (POD) which will be explained in details in Section 2. The second technique to determine ROM is the input/output model. This methodology use a transfer function, that typically receive input in structure modes and give output as generalized forces weighted by structural modes (Karpel, 1982).

We chose to study POD capacities in fluid structure interaction. In fact, this method was introduced in 1967 (Lumley, 1967) in fluid mechanic in order to extract coherent structures in a turbulent fluid flow. It has been intensively used since 90's in many application such as flows in a driven cavity (Cazemier et al., 1998) or in boundary layer (Sirovich et al., 1990).

In structure mechanics, POD is a recent investigation domain similar to modal analysis (Trindade et al., 2005; Sarkar et al., 2004; Sarkar et al., 2003; Epureanu et $a l ., 2004)$. There are few works in fluid structure interaction. In this paper the POD capacities are investigated in a two dimensional fluid structure interaction problem. Then the Burgers equation with moving boundaries is reduced in order to test the POD method on a simple case.

\section{The proper orthogonal decomposition (POD)}

\subsection{POD formulation}

In this section, the POD method is briefly introduced. A detailed methodology is already stipulated in literature (Lumley, 1967; Sirovitch, 1987; Aubry et al., 1988). 
The POD consists in finding a determinist function $\phi$, in a Hilbert space $H$, which gives the optimum representation of a random field $u(x, t) \in \Omega, \Omega \subset \mathbb{R}^{n}$, by solving the following maximization problem:

$$
\max _{\phi \in H} \frac{\left\langle(u, \phi)^{2}\right\rangle}{(\phi, \phi)}=\frac{\left\langle(u, \Phi)^{2}\right\rangle}{(\Phi, \Phi)}
$$

where $\langle\bullet\rangle$ denotes a statistic average operator, $(\bullet, \bullet)_{H}$ denotes the inner product of $H$ and $\|\bullet\|_{H}^{2}$ the associated norm. In the case of $H=L^{2}(\Omega)$, the maximization of problem [1] leads to the solving the following eigenvalue problem:

$$
\int_{\Omega} \mathcal{R}\left(x, x^{\prime}\right) \Phi\left(x^{\prime}\right) d x^{\prime}=\lambda \Phi(x)
$$

where $\mathcal{R}$ is the symetric spatial correlation tensor, defined non-negative:

$$
\mathcal{R}\left(x, x^{\prime}\right)=\left\langle u(\bullet, x) \otimes u\left(\bullet, x^{\prime}\right)\right\rangle .
$$

Moreover, if $\mathcal{R}$ is continuous, this operator

$$
\Phi \mapsto \int_{\Omega} \mathcal{R}\left(x, x^{\prime}\right) \Phi\left(x^{\prime}\right) d x^{\prime}
$$

is compact. Then Hilbert-Schmidt theory assures that there exists a set of positive eigenvalues $\left(\lambda_{i}\right)_{i \geq 1}$ which decrease to 0

$$
\lambda_{1}>\lambda_{2}>\cdots>\lambda_{i}>\cdots \text { and } \lambda_{i} \rightarrow 0
$$

And a set of eigenmodes $\left(\Phi_{i}\right)_{i \geq 1}$ which is a Hilbertien basis for $H$. Thus $u$ can be decomposed according the eigenmodes as:

$$
u(x, t)=\sum_{i=1}^{\infty} a_{i}(t) \Phi_{i}(x) \text { in } L^{2}(\Omega) \text { sense }
$$

where $a_{i}$ are the temporal coefficients.

$\left(\Phi_{i}\right)_{i \geq 1}$ are named modes. When the decomposition described by Equation [5] is introduced in the complete system with $N$ modes, a reduced system is obtained and the computational time is very small compared to the complete system.

\subsection{POD modes properties}

The spatial modes, $\left(\Phi_{i}\right)$ are orthogonals which can be normalised. So:

$$
\left(\Phi_{i}, \Phi_{j}\right)=\int_{\Omega} \Phi_{i}(x) \Phi_{j}(x) d x=\delta_{i j}
$$


and they respect boundaries conditions. In case of velocity fluid of incompressible flow, the POD modes respect $\operatorname{div} \Phi_{i}=0$

The temporal coefficients $a_{i}(t)$ result from the projection of $u$ in $\left(\Phi_{i}\right)$ basis:

$$
a_{i}(t)=\left(u(x, t), \Phi_{i}(x)\right)
$$

Moreover, they are uncorellated and the eigenvalues are the temporal average:

$$
\left\langle a_{i}(t) a_{j}(t)\right\rangle=\delta_{i j} \lambda_{i}
$$

The eigenvalue $\lambda_{i}$ is the energy captured by the mode $\Phi_{i}$. For a given $N$, the POD decomposition is the best energy decomposition which can be obtained.

\subsection{The snapshot POD}

Solving Equation [2] can be computationally intensive in higher dimensional problem. In order to minimize the computational times, snaphot method is used (Sirovitch, 1987).

Let $N_{m}$ be the node number, $n_{c}$, the component number and $\Phi$ a mode POD. If sampling of $M$ realisations $M<<N_{m} n_{c}$ of the flow is sufficient to describe the problem, then we search the temporal coefficients $a_{k}$ such as:

$$
\Phi(x)=\sum_{k=1}^{M} a_{k} u\left(x, t_{k}\right)
$$

With ergodicity hypothesis, the temporal average $\langle\bullet\rangle$ is a statistical one, and using the inner product of $L^{2}(\Omega)$, we have to solve the following eigenvalue problem:

$$
\sum_{i=1}^{M} \frac{1}{M}\left(u\left(t_{i}\right), u\left(t_{k}\right)\right) a_{k}=\lambda a_{i} \text { pour } i=1 \ldots M
$$

Hence the temporal coefficients $a_{i}$ are obtained by solving Equation [10] and the spatial modes by the Equation [9].

Using the classic or snapshot method depends on the data type. In the case of experimental data, the classic method is used. On the other hand, in the case of computational simulation with a significant grid and time limitation, the snapshot method is preferred. Note that the ergodicity hypothesis is debatable because a non-stationary problem is considered. Another solution would be the bi-orthogonal decomposition (Hemon et al., 2003), which does not need this hypothesis. 


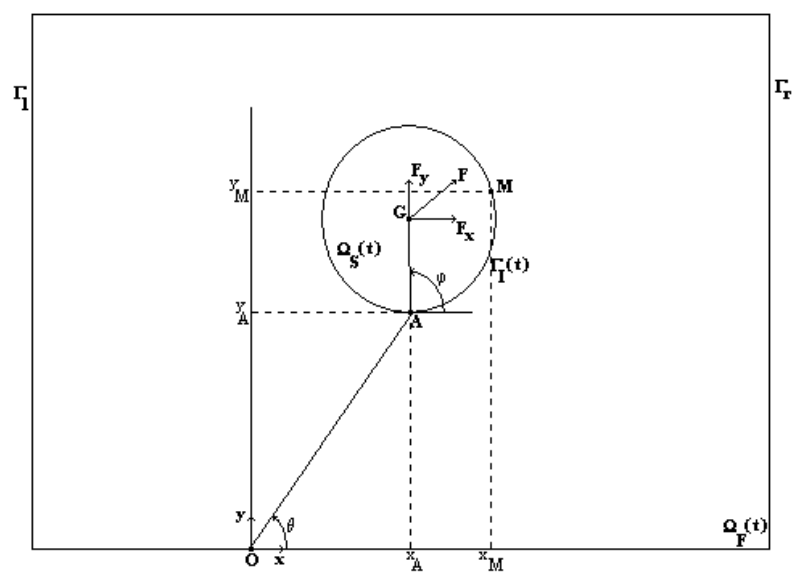

Figure 1. Schematic description of problem domain

\section{Fluid structure interaction application}

\subsection{ALE description of the Navier-Stokes equations}

POD was studied for a one and two dimensional case. We test a reducing method only on the one dimensional case, that is the reason that POD efficiency is viewed in Section 4 in association with reduced system study. In this section, global formulation is expressed for a two dimensional case. Consider a two-dimensional rigid body immersed in an incompressible fluid. Figure 1 shows a schematic description of the problem domain of interest, where $\Omega_{S}(t)$ is the domain occupied by the moving rigid body, of which the center of gravity is denoted by $G ; \Omega_{F}(t)$ is the moving spatial domain upon which the fluid motion is described; and $\Gamma_{I}(t)$ is the interface between $\Omega_{S}(t)$ and $\Omega_{F}(t)$. As the rigid body $\Omega_{S}(t)$ changes the position, the interface $\Gamma_{I}(t)$ moves accordingly.

The motion of the fluid is governed by the incompressible Navier-Stokes equations which are given as follows in the $A L E$ description (Donea, 2004; Nomura et al., 1992; Sarrate et al., 2001):

$$
\left\{\begin{array}{cccc}
\rho \frac{\partial u}{\partial t}+\rho(u-w) \cdot \nabla u & = & -\nabla p+\mu \Delta u & \\
\nabla \cdot u & = & 0 & \text { in } \Omega_{F}(t)
\end{array}\right.
$$

where $u$ is the fluid velocity, $w$ the fluid mesh velocity, $p$ is the fluid pressure, $\mu$ is the dynamic viscosity. 
The boundary, $\partial \Omega_{F}(t) \backslash \Gamma_{I}(t)$, is divided into two parts on which the following boundary conditions are specified:

$$
\begin{array}{ll}
u=U_{0} & \text { on } \Gamma_{l} \\
\sigma \cdot \mathbf{n}_{r}=0 & \text { on } \Gamma_{r}
\end{array}
$$

where $\mathbf{n}_{r}$ is the unit outward normal vector to $\Gamma_{r}$ and

$$
\sigma=-p I+\mu\left(\nabla u+{ }^{t} \nabla u\right)
$$

is the Cauchy stress tensor.

In this study $\Gamma_{l}$ and $\Gamma_{r}$ are assumed to be fixed in space. This assumption leads to

$$
w=0 \quad \text { on } \quad \Gamma_{r} \cup \Gamma_{l}
$$

Let the velocity on the moving interface $\Gamma_{I}(t)$ be denoted by $u^{I}$. This velocity is unknown, but

$$
u^{I}=w \quad \text { on } \Gamma_{I}(t)
$$

because of the non-slip condition on $\Gamma_{I}(t)$. In other words, we have the Lagrangian description on $\Gamma_{I}(t)$.

\subsection{Rigid body motion equation}

Let $V$ be the momentum field (similar to torsor) to represent the velocity field for the rigid body. Lets consider the Lie group and algebra formalism applied to the mechanics of body systems (Chevalier, 1994; Chevalier, 1984; Hamdouni et al., 1998). In this Lie algebra, lets denote $[\bullet, \bullet]$, the Lie bracket and $\langle\bullet, \bullet\rangle$, the non-degenerated, symmetric, bi-linear form which is the co-momentum.

Consider the generalized inertia operator of $\Omega_{S}(t), \mathcal{H}$, which contains all information about the body inertia (center of inertia, mass, inertia matrix) explained in detail in (Chevalier, 1984). In this formalism, we express the rigid solid equation with the following expression:

$$
\mathcal{H} \frac{d V}{d t}+[V, \mathcal{H} V]=\mathcal{F}_{s}+\mathcal{F}_{F}
$$

In our application, we consider a planar motion of a system of rigid bodies, i.e. rigid disc of mass $M$ and radius $r$, linked by rigid bar of mass $m$ and length $l$ to the lower boundary. In this two-dimensional case, the motion of this system has two degrees of freedom; the two rotational displacements $\theta$ and $\varphi$ defined at the points $O$ 
and $A$ respectively as shown in Figure 1. In this case, the equation of motion of the system is written as

$$
\mathbf{M}(\theta, \varphi) \ddot{\Psi}+\mathbf{C}(\theta, \varphi, \dot{\theta}, \dot{\varphi}) \dot{\Psi}=A(\theta, \varphi) \mathbf{F}+\mathbf{b}(\theta, \varphi)
$$

with appropriate initial conditions. Where $\Psi=(\theta, \varphi)^{t}$ and $\mathbf{F}=\left(F_{x}, F_{y}, \mathcal{M}_{G}\right)^{t} . \mathbf{M}$ and $\mathbf{C}$ are the mass and the damping matrices respectively. $\mathbf{F}$ and $\mathbf{b}$ contain the fluid force and the momentum resultants and gravity force respectively. The fluid force and the momentum resultants are given by the following equations

$$
F_{f}=\int_{\Gamma_{I}} \sigma \cdot n_{s} d \gamma \text { and } \mathcal{M}_{G}=\int_{\Gamma_{I}} \overrightarrow{G M} \times \sigma \cdot n_{s} d \gamma
$$

where $n_{s}$ is the unit outward normal vector to $\Gamma_{I}$

\subsection{Rigid body displacement}

Assume that at time $t=t_{n}, \theta(t)=\theta_{n}$ and $\varphi(t)=\varphi_{n}$. In this case, the coordinates of the points $A_{n}=A\left(t_{n}\right)$ and $G_{n}=G\left(t_{n}\right)$ are written respectively as,

$$
\left\{\begin{array}{l}
x_{A}\left(t_{n}\right)=x_{A}^{(n)}=l \cos \theta_{n} \\
y_{A}\left(t_{n}\right)=y_{A}^{(n)}=l \sin \theta_{n}
\end{array}\right.
$$

and

$$
\left\{\begin{array}{l}
x_{G}\left(t_{n}\right)=x_{G}^{(n)}=r \cos \varphi_{n}+x_{A}^{(n)} \\
y_{G}\left(t_{n}\right)=y_{G}^{(n)}=r \sin \varphi_{n}+y_{A}^{(n)}
\end{array}\right.
$$

For any point $M_{n}=M\left(t_{n}\right)=\left(x_{n}, y_{n}\right)$ on $\Gamma_{I}\left(t_{n}\right)$, the vector $\overrightarrow{A_{n} M_{n}}$ can be written as,

$$
\overrightarrow{A_{n} M_{n}}=\mathbf{T}\left(t_{n}\right)+\mathbf{R}\left(t_{n}, \varphi_{n}-\theta_{n}\right) \cdot \overrightarrow{A_{n-1} M_{n-1}}
$$

where the translation displacement $\mathbf{T}\left(t_{n}\right)$ of the rigid body is given by,

$$
\mathbf{T}\left(t_{n}\right)=\overrightarrow{A_{n-1} A_{n}}=\left(\begin{array}{c}
x_{A}^{(n)}-x_{A}^{(n-1)} \\
y_{A}^{(n)}-y_{A}^{(n-1)}
\end{array}\right)
$$

and the rotation displacement $\mathbf{R}\left(t_{n}\right)$ around the point $A_{n}$ is given by,

$$
\mathbf{R}\left(t_{n}, \varphi_{n}-\varphi_{n-1}\right)=\left(\begin{array}{cc}
\cos \left(\varphi_{n}-\varphi_{n-1}\right) & -\sin \left(\varphi_{n}-\varphi_{n-1}\right) \\
\sin \left(\varphi_{n}-\varphi_{n-1}\right) & \cos \left(\varphi_{n}-\varphi_{n-1}\right)
\end{array}\right)
$$


Then, the coordinates of $M_{n}$ are given by,

$$
\left\{\begin{aligned}
x_{n}= & x_{A}^{(n)}+\left(x_{n-1}-x_{A}^{(n-1)}\right) \cos \left(\varphi_{n}-\varphi_{n-1}\right) \\
& -\left(y_{n-1}-y_{A}^{(n-1)}\right) \sin \left(\varphi_{n}-\varphi_{n-1}\right) \\
y_{n}= & y_{A}^{(n)}+\left(x_{n-1}-x_{A}^{(n-1)}\right) \sin \left(\varphi_{n}-\varphi_{n-1}\right) \\
& +\left(y_{n-1}-y_{A}^{(n-1)}\right) \cos \left(\varphi_{n}-\varphi_{n-1}\right)
\end{aligned}\right.
$$

\subsection{Mesh motion description}

The mesh velocity vector $w$ may be arbitrarily specified though it has to satisfy the following conditions on boundaries,

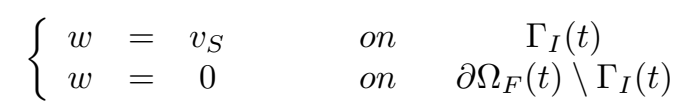

where $v_{S}$ is the velocity vector of the rigid body nodes on the interface.

In this study, the mesh velocity in the fluid domain is determined by solving the following equation

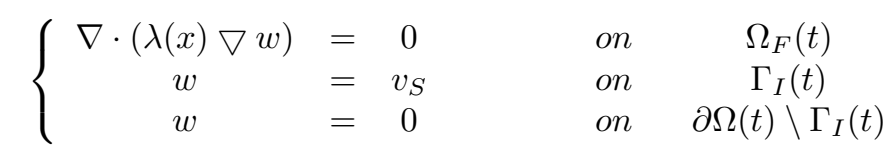

where $\lambda(x)$ is judiciously chosen to control the mesh deformation. In this case, $\lambda(x)$ is taken equal to 1 for all $x \in \Omega_{F}(t)$.

\subsection{Fluid-rigid body interaction algorithm}

The solid Equation [16] is solved by coupling the finite difference and GaussSeildel iterative methods. It firstly transformed to the following system of equations.

$$
\left\{\begin{aligned}
\dot{Y} & =A \mathbf{F}+\mathbf{b}+\left(\frac{d \mathbf{M}}{d t}-\mathbf{C}\right) \dot{\Psi} \\
\mathbf{M} \dot{\Psi} & =Y
\end{aligned}\right.
$$

Then, from initial conditions on $\Psi$ and $\dot{\Psi}$, the solution is obtained through iterations until a fixed error of accuracy is reached.

Implicit Euler method is used to a time discretization of fluid Equations [11] and finite element method to a spatial discretization. 
To solve the coupling equations, following explicit scheme is used (Abouri et al., 2004).

Suppose that time $t=t_{n}$, the fluid velocity and pressure fields, the rigid body displacement and position are known. The time step $(\triangle t)$ of the Navier-Stokes equations solver is same the rigid body equation.

1. The body Equations [26] are solved in order to compute the displacement velocity $v_{S}$ at time $t_{n+1}$. Then, the position of $\Gamma_{I}\left(t_{n+1}\right)$ can be determined by solving the Equation [23] for all the nodes on $\Gamma_{I}\left(t_{n}\right)$.

2. The mesh velocity Equation [25] is solved and the velocity $w^{(n+1)}$ of the fluid nodes displacement at time $t_{n+1}$ is determined.

3 . The rigid body and the fluid nodes are moved at the predicted position by solving the equation $x^{(n+1)}=w^{(n+1)} \Delta t+x^{n}$ for all the mesh nodes. Then the fluid domain $\Omega\left(t_{n+1}\right)$ is defined.

4. The fluid Equations [11] are solved in the domain $\Omega_{F}\left(t_{n+1}\right)$.

5. The fluid forces acting on the rigid body are computed using Equations [17].

This explicit algorithm is easy to implement, but it is only of order one and requires a small time step to its stability. An implicit scheme can also be used.

\subsection{Numerical application}

To illustrate this algorithm for fluid-rigid body interaction, the fluid domain size is taken equal to $21 \times 12 \mathrm{~m}, r=0.5 \mathrm{~m}, l=5.5 \mathrm{~m}, M=50 \mathrm{~kg}, \mathrm{~m}=1 \mathrm{~kg}, U_{0}=1 \mathrm{~m} / \mathrm{s}$, $\theta_{0}=\varphi_{0}=90^{\circ}$ and $\triangle t=10^{-2} \mathrm{~s}$.

Two kinds of finite elements are used in spatial discretization of the fluid domain. Around the rigid body, the mixed Crouzeix-Raviart element and in the rest of the domain the $\Phi_{2} / \boldsymbol{P}_{1}$-discontinuous element are used. The mesh contains 4120 nodes and 1036 elements as shown in Figure 2. The computations are achieved using Castem code (CEA, 2005) during 5.55s.

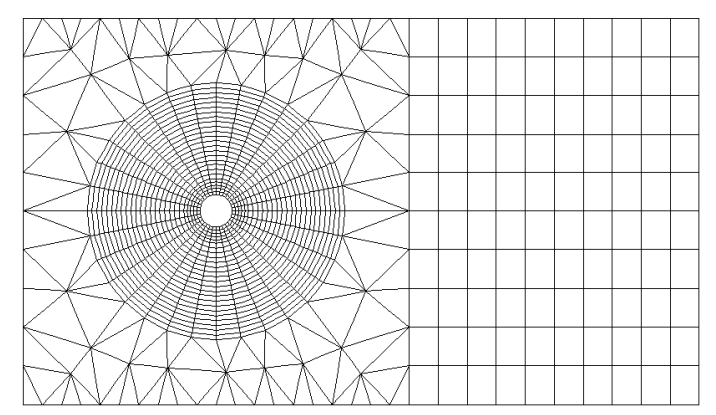

Figure 2. Fluid mesh 


\subsection{POD application}

The computational result of Equation [11] define the velocity field on the timevariant grid. In search of spatial POD modes on this field, we could not determine domain where they would be defined. Thus we could not define a scalar product. We do not search POD modes for only the velocity fluid flow but for all the velocity field in the $\Omega$ domain $\left(\Omega=\Omega_{F}(t) \cup \Omega_{S}(t)\right)$.The solution at each time step is interpolated from the time-variant grid to a fixed uniform grid by an inverse distance interpolation method (Zhang et al., 2003). To obtain fluid and structure domain, a characteristic function $X_{\Omega_{j}}, j \in\{F, S\}$ of each domain, $\Omega_{j}, j=F, S$, is used:

$$
\begin{aligned}
& X_{\Omega_{j}}(x, t)= \begin{cases}1 & \text { if } x \in \Omega_{j}(t) \\
0 & \text { if } x \notin \Omega_{j}(t)\end{cases} \\
& \forall u \in \Omega \quad \int_{\Omega} \nabla u \cdot X_{\Omega_{j}}=\left.u\right|_{\Gamma_{I}}
\end{aligned}
$$

Thus we define a velocity field $v$ on $\Omega$ :

$$
v(x, t)=\left\{\begin{array}{l}
v_{f}(x, t) \text { if } x \in \Omega_{F}(t) \\
v_{s}(x, t) \text { if } x \in \Omega_{S}(t)
\end{array}\right.
$$

Or

$$
v(x, t)=v_{f}(x, t) X_{\Omega_{F}}(x, t)+\left(1-X_{\Omega_{F}}(x, t)\right) v_{s}(x, t)
$$

We use $v_{f}(x, t)$ on a rigid grid, thus we use Eulerian formulation for the fluid in weak formulation.

\subsection{POD efficiency in fluid structure interaction}

In this section the POD efficiency is tested to reconstruct a velocity field in a fluidstructure interaction problem. An interpolation of the solution to a rigid grid (cf. Section 3.7) is done and snapshot POD on this new velocity field is used. Table 1 shows the kinetic energy contribution of each POD mode contained by each eigenvalue:

$$
\sum_{k=1}^{i} \lambda_{k} / \sum_{k=1}^{N} \lambda_{k}
$$


Table 1. Kinetic energy contribution for the five first modes

\begin{tabular}{|c|c|c|}
\hline mode $i$ & eigenvalue $\lambda_{i}$ & \% energy \\
\hline 1 & 254 & 99.837 \\
2 & 0.343 & 99.9722 \\
3 & $5.553 e-2$ & 99.994 \\
4 & $1.100 e-2$ & 99.998 \\
5 & $2.674 e-3$ & 99.999 \\
\hline
\end{tabular}

To find POD modes by snapshot method, we have temporal coefficients and POD modes. We can evaluate the truncated velocity field by using the truncated POD basis functions like in Equation [5]:

$$
U(x, t)=\sum_{n=1}^{N} a_{n}(t) \Phi_{n}(x)
$$

where

$$
U=\left\{\begin{array}{l}
u \\
v
\end{array}\right\} \text { and } \Phi_{n}=\left\{\begin{array}{c}
\Phi_{u}^{n} \\
\Phi_{v}^{n}
\end{array}\right\}
$$

To evaluate the computational efficiency of the solution obtained by the POD method, the following $L^{2}$ error norm function is defined:

$$
\operatorname{Res}(N)=\frac{\left\|U-U_{N}\right\|_{L^{\infty}\left(0, T ; L^{2}(\Omega)\right)}}{\|U\|_{L^{\infty}\left(0, T ; L^{2}(\Omega)\right)}}
$$

Figure 3 shows that with four POD modes the error is less than $1 \%$ for the reconstructed solution. This first analysis on a two dimensional case gives good result.

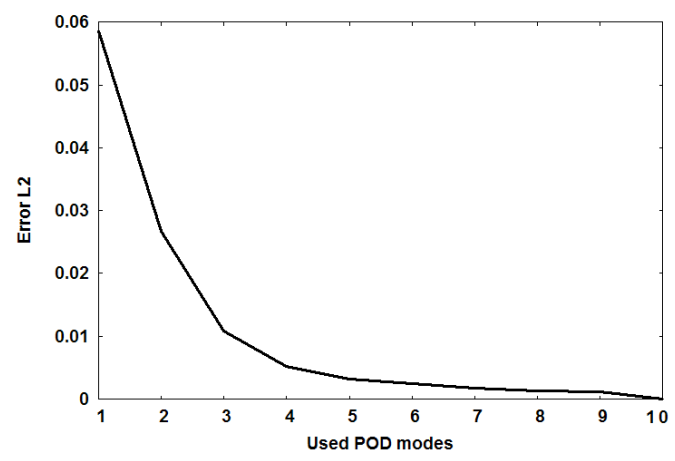

Figure 3. Error nom of velocity versus number of $P O D$ modes 
We can hope reducing the dynamical system, in the same temporal interval,with less than ten modes whereas the original solution was obtained with 8140 degrees of freedom model. The eigenmodes of the velocity field are illustrated of Figure 4 and Figure 5.
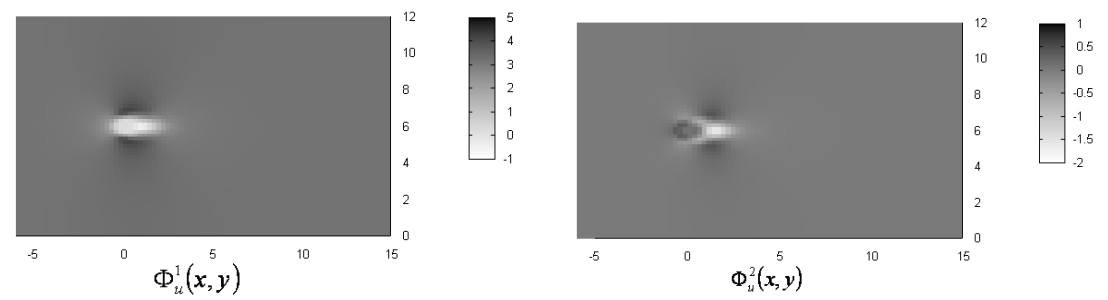

Figure 4. First component of POD basis functions 1 and 2
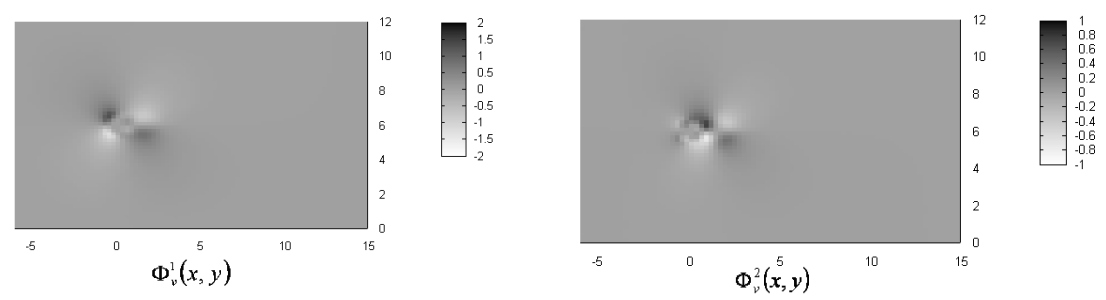

Figure 5. Second component of POD basis functions 1 and 2

We can see in this figures that POD modes swirls where the rigid body move during simulation.

\section{Reduction Application of Burgers equation coupled with ressort Equation}

In this section, we will introduce a methodology to reduce dynamical system in fluid structure interaction and test them on the case of Burgers equation in a moving boundaries. We call $\Omega_{F}(t)$ the fluid domain at $t$ time, $\Omega_{S}(t)$ the solid domain, $u$ a fluid velocity field, $w$ the fluid grid velocity and $x$ a coordinates in $\Omega$. 


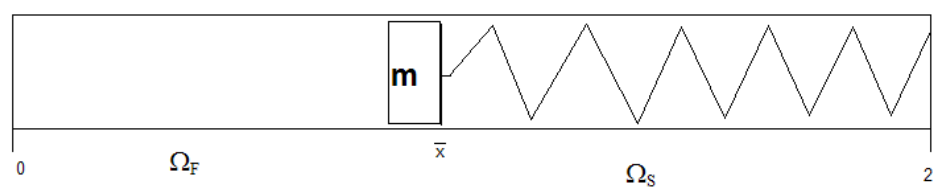

Figure 6. Shematic of the fluid and structure domain

The Burgers equation in ALE formulation is solved in $\Omega_{F}(t)$ on set time interval $[0, T]$ :

$$
\begin{cases}\frac{\partial u}{\partial t}+(u-w) \frac{\partial u}{\partial x}-\nu \frac{\partial^{2} u}{\partial x^{2}}=0 & \text { on } \Omega_{F}(t) \\ u(0, t)=0 & \\ u(\bar{x}, t)=\frac{d \bar{x}}{d t} & \text { on } \Omega_{F}(0)\end{cases}
$$

$\bar{x}$ is the interface coordinates, which is solved by the equation of the spring mass system:

$$
m \ddot{\bar{x}}+K\left(\bar{x}-x_{0}\right)+b \dot{\bar{x}}=F_{F}
$$

where $x_{0}$ is the "at rest" length of spring, $b$ the damping constant, $K$ the spring stiffness, $m$ the mass of the block and $F_{F}$ the fluid force. We choose $x_{0}=0.95 m, b=$ $0.10 N . s . m^{-1}, K=39.5 \mathrm{~N} . \mathrm{m}^{-1}, m=0.5 \mathrm{~kg}$. This system is solved by NewtonRaphson algorithm and in next section the solution is compared with them obtained from reduced system.

Using an inverse distance interpolation method, a velocity field $v$ on a fixed uniform grid is obtained like defined in Section 3.7. Thus Eulerian formulation for the fluid with $v f$ is used for obtain reduced system.

\subsection{Weak formulation}

The weak formulation is expressed for a three dimensional case of fluid-rigid body interaction.

Let $v^{*}$ a virtual velocity field of fluid such as $\operatorname{div} v^{*}=0$ and $V^{*}$ a virtual moment field for structure. 
We choose $v^{*}$ and $V^{*}$ consistently, acceptable in kinematic sense, and we assume that there is no slip at fluid-solid interface and action-reaction principle

$$
\left\langle\mathcal{F}_{F}, V^{*}\right\rangle+\int_{\Gamma_{I}}\left(-p I+\tau\left(v_{f}\right)\right) \cdot n \cdot v^{*} d \gamma=0
$$

where $I$ is identity amtrix, $\tau$ the viscous stress tensor $\tau\left(v_{f}\right)=2 \mu \mathcal{D}\left(v_{f}\right)$ and

$$
\mathcal{D}\left(v_{f}\right)=\frac{1}{2}\left(\nabla v_{f}+{ }^{t} \nabla v_{f}\right)
$$

The weak formulation can be written:

$$
\begin{aligned}
\left(\rho \frac{\partial v_{f}}{\partial t}+\rho v_{f} \cdot \nabla v_{f}, v^{*}\right)- & \left(\mu \triangle v_{f}, v^{*}\right)+\left\langle\mathcal{H} \frac{d V}{d t}, V^{*}\right\rangle \\
+ & \left\langle[V, \mathcal{H} V], V^{*}\right\rangle=\left\langle\mathcal{F}_{s}, V^{*}\right\rangle
\end{aligned}
$$

\subsection{Dynamic system reduction}

A set of the $N$ first POD modes $\left\{\left(\Phi_{i}\right)_{i=1 . . N}\right\}$ are used such as

$$
\sum_{k=1}^{N} \lambda_{k} / \sum_{k=1}^{\infty} \lambda_{k} \geq 99.99 \%
$$

The field $v$ is searched on its truncated formulation:

$$
v_{N}(x, t)=\sum_{n=1}^{N} a_{n}(t) \Phi_{n}(x)
$$

Only the fluid dynamical system is reduced because in case of rigid body the dynamical system is not expensive to compute. Let use $v^{*}=X_{\Omega_{F}} \Phi_{n}$ and using POD modes that are orthonormal in $L^{2}(\Omega)$ sense, the weak formulation becomes:

For $n=1 . . N$ :

$$
\left\{\begin{array}{l}
\frac{d a_{n}}{d t}+\sum_{m=1}^{N} \sum_{p=1}^{N} \mathcal{B}_{n m p} a_{m} a_{p}+\sum_{m=1}^{N} \mathcal{A}_{n m} a_{m}=\int_{\Omega} \frac{\partial v_{s}}{\partial t} \cdot \Phi_{n} d x \\
\mathcal{H} \frac{d V}{d t}+[V, \mathcal{H} V]=\mathcal{F}_{s}+\mathcal{F}_{F} \text { in } \Omega_{S}(t)
\end{array}\right.
$$

where

$$
\begin{aligned}
& \mathcal{A}_{n m}=\mu \int_{\Omega} \nabla \Phi_{m} \cdot \nabla \Phi_{n} X_{\Omega_{F}} d x+\left.2 \mu\left(\nabla \Phi_{m} \cdot \Phi_{n}\right)\right|_{\Gamma_{I}} \\
& \mathcal{B}_{n m p}=\int_{\Omega} \Phi_{m} \cdot \nabla \Phi_{p} . \Phi_{n} X_{\Omega_{F}} d x
\end{aligned}
$$




\subsection{Energy contribution of POD modes}

We apply POD method defined in Section 2.3. The first six POD modes have more than $99.99 \%$ of the kinetic energy ( $c f$. Table 2). Next, the reduced system [37] is solved with $N=6$.

Table 2. Kinetic energy contribution for the five first modes

\begin{tabular}{|c|c|c|}
\hline mode $i$ & eigenvalue $\lambda_{i}$ & \% energy \\
\hline 1 & 18.255 & 91.648 \\
2 & 1.515 & 99.256 \\
3 & $8.393 e-2$ & 99.677 \\
4 & $6.079 e-2$ & 99.982 \\
5 & $2.347 e-3$ & 99.994 \\
6 & $7.804 e-4$ & 99.999 \\
\hline
\end{tabular}

The low-order truncation of the POD basis inhibits generally all the transfers between the large and the small (unresolved) scales of the fluid flow. Consequently, to recover the effects of the truncated modes, that is generally of the small scales, we use a "eddy viscosity" (Aubry et al., 1988; Podvin, 2001). In fact the viscosity for the mode $i$ is multiplied by $(1+i * 0.001)$.

Reducing the system with only six modes, give a good result with an error less $4 \%$, illustrated in Figure 7. Figure 8 shows that the error is relatively not very significant.
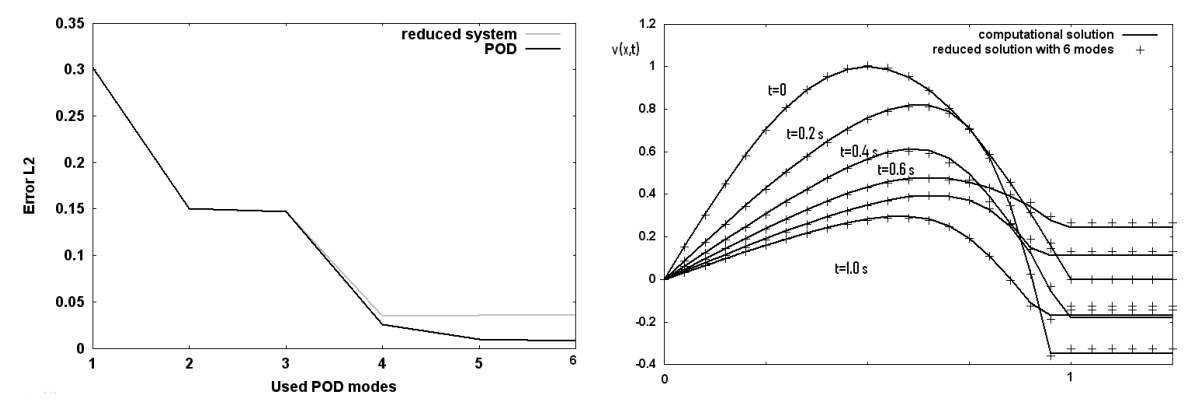

Figure 7. $L 2$ Error norm of velocity versus Figure 8. Solution of the reduced system number of POD modes

The reconstructed temporal modes resulting from reduced system and those are resulting from snapshot method are compared in Figure 9. The difference is very small between them. The difference comes from computational approximation of modes and derivatives at interface. Indeed, the interface position is between nodes and we need to know for example $\Phi_{i}(\bar{x})$ by interpolating on the two first nodes whose are the nearest. 
REMN - 16/2007. Fluid structure interaction
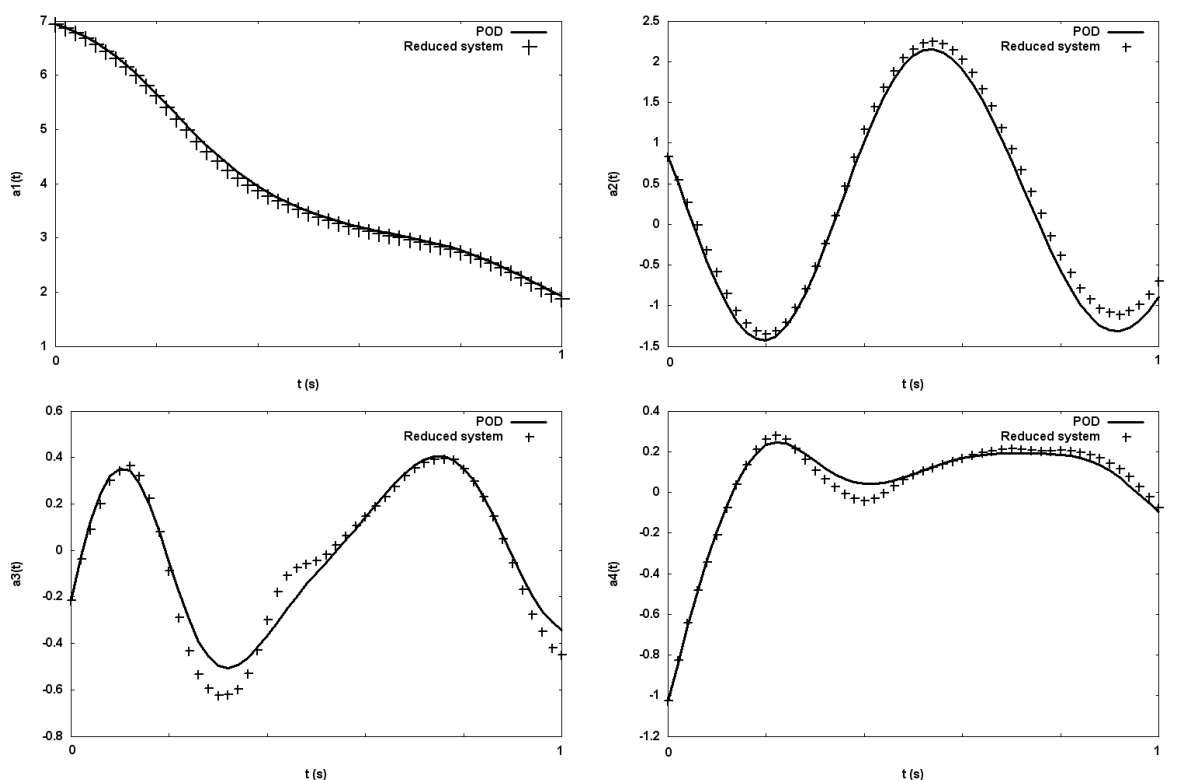

Figure 9. Temporal coefficients $a_{1}, a_{2}, a_{3}, a_{4}$
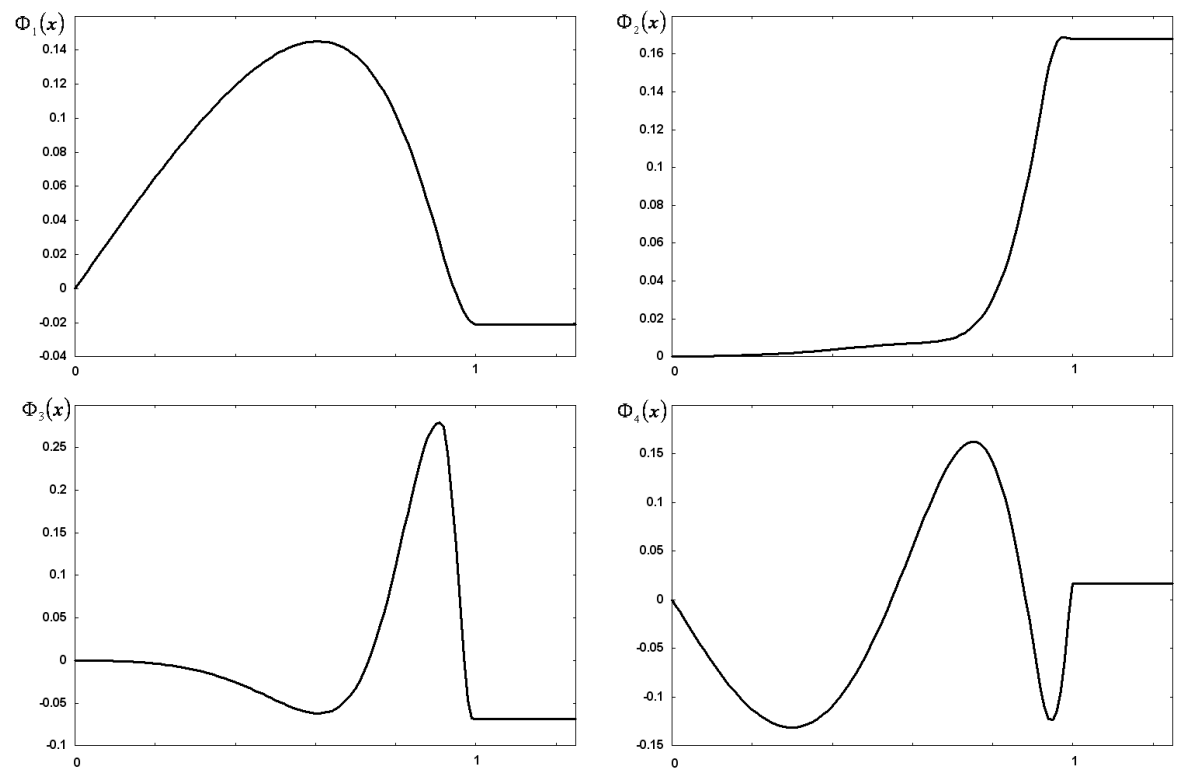

Figure 10. 4 first POD basis functions 
The first mode which is consistent with the mean velocities properties, capture at least $99 \%$ of the kinetic energy in all case which have tested. The second is similar to the interface moving. The another modes are used to keep velocity varitions in the fluid. We can see that where domain is always structured the POD modes are constant. In fact we supposed that the body is a rigid structure.

\section{Conclusion}

We have presented POD methodology and its application in fluid-structure interaction (FSI). The principal difficulties to apply it was the spatial properties of POD modes and the fact that in FSI the fluid domain moves in time. Thus, we chose to study global velocity field on a referential domain. To apply POD for global velocity field, the characteristic function of fluid is introduced. Tests of POD efficiency on one and two dimensional cases for global velocity field yield good results. Then a method to reduce dynamical system in rigid body fluid interaction is developed and tested on the one dimensional case of Burgers equation in moving domain. Conformation between computational results from Newton-Raphson and thus obtained by reduced system are found.

\section{References}

Abouri D., Parry A., Hamdouni A., " Stable fluid rigid body interaction algorithm : application to industrial problems", 8th International Symposium on Emerging Technologies for Fluids, Structures, and Fluid-Structure Interaction, ASME/JSME, San Diego California USA, p. 25-29, July, 2004.

Aubry N., Holmes P., Lumley J., Stone E., “ The dynamics of coherent structures in the wall region of a turbulent boundary layer", Journal of Fluid Mechanics, vol. 192, p. 115-173, 1988.

Baker M., Mingori D., Goggin P., “ Approximate subspace iteration for constructing internally balanced reduced order model of unsteady aerodynamic systems", AIAA Paper, 1996.

Cazemier W., Verstappen R., Veldman A., " Proper orthogonal decomposition and lowdimensional models for driven cavity”, Physics of fluids, vol. 10.7, p. 1685-1699, 1998.

CEA, www-cast3m.cea.fr, Technical report, CEA, 2005.

Chevalier D., " Groupe de Lie et mécanique des systèmes de corps rigides”, MacGraw-Hill, 1984.

Chevalier D., “ Lie groups and multibody Dynamics formalism”, EUROMECH, Prague, 1994.

Donea J., “ Arbitrary Lagrangian-Eulerian Methods", Encyclopedia of Computational Mecanics, 2004.

Dowell E. H., Hall, Kenneth C., “ Modelling of Fluid-Structure Interaction”, Annuals Review of Fluid Mechanics, vol. 33, p. 445-90, 2001.

Epureanu B. I., Tang L. S., Païdoussis M. P., " Coherent structures and their influence on the dynamics of aeroelastic panels”, International Journal of Non-Linear Mechanics, vol. 17, n 6, p. 977-991, 2004. 
Hamdouni A., Taibi N., “Kane's formalism and Lie group theory”, Mechanics Research Communications, vol. 25, $\mathrm{n}^{\circ}$ 4, p. 385-393, 1998.

Hemon P., Santi F., "Applications of biorthogonal decompositions in fluid-structure interactions", Journal of fluids and Structures, vol. 17, p. 1123-1143, 2003.

Karpel M., " Design for active flutter suppression and gust alleviation using state-space aeroelastic modelling”, J. Aircraft, vol. 19, n 3, p. 221-27, 1982.

Lumley J., " The structure of inhomogeneous turbulent flows", Atmospheric Turbulence and Radio Wave Propagation, vol. In A.M. Yaglom and Tararsky, p. 166-178, 1967.

Mahajan A., Bakhle M., Dowell E., “ A new method for aeroelastic stability analysis of cascades using nonlinear, time marching CFD solvers”, AIAA Paper, 1994.

Nomura T., Hughes T., “ An arbitrary Lagrangian-Eulerian finite element method for interaction of fluid and rigid body", Computer Methods in Applied Mechanics and Engineering, vol. 95, p. 115-138, 1992.

Podvin B., " On the adequacy of the ten-dimensional model for the wall layer", Phys. Fluids, vol. 13, p. 210-224, 2001.

Romanowski M., Dowell E., " Reduced order Euler equation for unsteady aerodynamics flow: numerical techniques", AIAA Paper, 1996.

Rule J., Cox D., Clark R., “ Aerodynamic model reduction through balanced realisation”, AIAA J., 2000.

Sarkar A., Paidoussis M. P., “ A Compact Limit Cycle Oscillation Model for a Cantilevered conveying Fluid", Journal of Fluids and Structures, vol. 17, p. 525-535, 2003.

Sarkar A., Paidoussis M. P., " A cantilever conveying fluid: coherent modes versus beam modes”, International Journal of Non-Linear Mechanics, vol. 39, n 3, p. 467-481, 2004.

Sarrate J., Huerta A., Donea J., “ Arbitrary Lagrangian-Eulerian formulation for fluid-rigid body interaction”, Computer Methods in Applied Mechanics and Engineering, vol. 190, n 6, p. 3171-3188, 2001.

Sirovich L., Ball K., Keefe L., "Planes waves and structure in turbulent channel flow”, Physics of fluids A2, vol. 12, p. 2217-2226, 1990.

Sirovitch L., " Turbulence and the dynamics of coherent structures, Part I : Coherent strucures, Part II : Symmetries and transformations, Part III : Dynamics and scaling", Quarterly of Applied Machanics, vol. 45, $\mathrm{n}^{\circ}$ 3, p. 561-590, 1987.

Trindade M. A., Wolter C., Sampaio R., “ Karhunen-Loève decomposition of coupled axial/bending vibrations of beams subject to impacts", Journal of Sound and Vibration, vol. 279, $\mathrm{n}^{\circ}$ issues 3-5, p. 1015-1036, 2005.

Zhang B., Lian T., Shyy W., " Proper orthogonal decomposition for three-dimensional membrane wing aerodynamics", AIAA Paper, vol. 3917, p. 1-15, 2003. 